\title{
Observation Units: Definition, History, Data, Financial Considerations, and Metrics
}

\author{
Jason D. Napolitano $\cdot$ Inderpreet Saini
}

Published online: 21 January 2014

(c) Springer Science+Business Media New York 2014

\begin{abstract}
Observation units are dedicated units built to provide efficient protocol-based care to patients with welldefined diagnoses or presenting symptoms such as chest pain, asthma, and congestive heart failure. Only approximately one-third of US hospitals currently have an observation unit. The efficiency which results from use of such units may improve bed capacity in individual hospitals and also provide great national cost savings as care is delivered safely in less time with use of fewer resources. Starting a unit can be challenging and there is much to be considered when selecting a medical director for the unit, choosing the diagnoses suitable for a given unit, and deciding on metrics to help monitor the performance of an observation unit.
\end{abstract}

Keywords Observation - Observation unit - Clinical decision unit - Emergency and Hospital Medicine

\section{Introduction}

According to the Centers for Medicare and Medicaid Services (CMS) definition: "Observation care is a well-defined set of specific, clinically appropriate services, which include ongoing short term treatment, assessment, and reassessment before a decision can be made regarding whether patients will require further treatment as hospital inpatients or if they are able to be discharged from the hospital. Observation services are commonly ordered for patients who present to the emergency department and who

J. D. Napolitano $(\varangle)$. I. Saini

UCLA Department of Medicine, David Geffen School of

Medicine at UCLA, 757 Westwood Plaza, Suite 7501, Los

Angeles, CA 90095, USA

e-mail: Jnapolitano@mednet.ucla.edu then require a significant period of treatment or monitoring in order to make a decision concerning their admission or discharge [1]." When observation status was first created it was thought that most patients would be observed for less than $24 \mathrm{~h}$.

Put simply, observation care equates to observing patients by use of serial clinical examinations to see if either:

- their medical condition can be brought under enough control to make it safe for them to be safely discharged to home; or

- enough diagnostic certainty can be met (for example for a patient with chest pain) to know the patient will be at minimal risk of adverse outcome after discharge.

Observation status was also initially believed to be most applicable to specific syndromes and conditions such as chest pain, asthma exacerbation, and heart failure exacerbation. For example, a patient with asthma exacerbation who had not improved adequately after several hours of treatment in the emergency department could be placed on observation to see whether improvement occurred after up to 24 more hours of inhaled bronchodilators and systemic corticosteroids. If improvement occurred he could be sent home. If he failed to improve or his condition worsened he could be admitted as an inpatient. Over time, observation status has become acceptable for almost any condition that may be managed over a short period of time in the hospital. Observation stays are becoming longer-a recent study at an academic medical center showed that fewer than onethird of cases of adult general medicine observation ended within $24 \mathrm{~h}$ and over one quarter of observation stays lasted longer than $48 \mathrm{~h}$.

Medicare does not specify where observation services should be delivered. Practices differ between hospitals with 
some observing patients in the emergency department, some placing patients on observation status in standard hospital rooms, and some placing these patients in special stand-alone observation units. Although few randomized prospective studies have compared admission of observation patients to dedicated observation units versus standard hospital wards, the comparisons that have been made seem to suggest better efficiency with similar patient outcomes when patients are admitted to dedicated observation units [2-5]. Currently approximately one-third of hospitals have a dedicated observation unit.

\section{Trends in Observation Care}

Both the number and percentage of patients admitted to observation status in the US have increased drastically over the last decade. Whereas $3 \%$ of patients in hospitals were on observation status in $2006,8 \%$ of patients were on observation status in $2011\left[6^{\bullet}, 7\right]$. In a recent review of observation stays at a large academic medical center, $25 \%$ of adults under general medical care in the hospital were under observation $[8 \bullet \bullet$.

The number of patients placed on outpatient observation despite being cared for in a hospital is rising, and not because more patients are being hospitalized to observe their responses to treatment. Instead, the increase in observation visits represents a shift in labeling patients as observation status rather than inpatient status, brought about by Medicare's strict criteria (usually based on Milliman or InterQual criteria) of what makes a patient sick enough to warrant hospital admission [9]. Because Medicare will not reimburse hospitals for inpatient charges for patients who should have been placed on observation, hospitals are prioritizing labeling and billing for observation care so that at least some payment is received for care rendered. Appropriate labeling of patients has begun to take on more urgency with the financing of recovery audit contractors (RAC) by Medicare. RAC auditors are commissioned to retrospectively find "overpayments" made to hospitals on the basis of documentation found in patient charts. Much of the money reclaimed for overpayments is for patients that hospitals deemed inpatients but who did not meet objective inpatient criteria. A three-year RAC demonstration project published in 2008 showed Medicare was able to take back almost one billion dollars that had been previously paid to hospitals [10].

A new Medicare rule which is being referred to as the "two midnight rule" went into effect October 1st 2013. This rule states that a patient whose hospitalization spans a time frame over two midnights should qualify as an inpatient for the purpose of billing of hospitals. Medicare and RAC auditors are studying charts to see which patients should be appropriately categorized as inpatients [11]. It is unclear at this point whether this new rule (which will not be enforced for 90 days after it becomes active) will ultimately lead to more or less patients being cared for under a designation of observation status while in the hospital.

\section{Setting up an Observation Unit}

Creating an observation unit will require approval of finance from hospital management because initial costs are substantial. These costs include construction of the unit, purchase of new monitors and other equipment, and hiring and training of staff. Many considerations are appropriate when choosing a medical director and the types of physician for staffing for a given unit (Table 1). In general, emergency medicine physicians have traditionally directed such units but hospitalists are now also being asked to direct or co-direct units. The latest available survey data reveal that $56 \%$ of US observation units are administratively managed by emergency department staff [12•]. A single study comparing emergency department-centered chest pain observation units with in-hospital chest-pain observation units suggests that treating such patients in an emergency department unit might be more cost effective [13]. An interesting and apparently unexplained finding in that study was that only $37 \%$ of patients presenting to the emergency department with chest pain were placed under observation when the emergency department observation unit was being used compared with $69 \%$ of patients with chest pain being placed under observation when the inhospital observation unit was being used. The choice of a medical director could be very dependent on the local patient population and the preferences of locally practicing physicians. For example, if most patients presenting to a particular unit will have stays that span more than one calendar day for conditions such as pneumonia, pyelonephritis, cellulitis, and sickle cell pain a hospitalist staffing model might result in greater continuity of care for patients, and free emergency medicine physicians for the care of more acutely ill patients. Of course, shared responsibility for a unit between emergency medicine and hospital medicine could lead to unique opportunities for enhanced communication and joint learning among teams.

Most observation units are run by attending physicians without the support of residents or medical students [14]. It could be argued that seasoned clinical judgment is needed to determine whether it is appropriate for patients to be cared for in an observation unit, and that substantial time is invested in learning inclusion and exclusion criteria for the unit. At UCLA, the observation unit was staffed for five years with resident physicians. Although the unit ran well and many trainees considered the unit a place to refine their 
Table 1 Comparison of possible advantages of emergency medicine or hospital medicine directorship of an observation unit

\begin{tabular}{|c|c|}
\hline Emergency medicine & Hospital medicine \\
\hline Shorter length of stay & $\begin{array}{l}\text { Greater variety of acceptable } \\
\text { diagnoses }\end{array}$ \\
\hline $\begin{array}{l}\text { More cost effective (per } \\
\text { individual patient) }\end{array}$ & $\begin{array}{l}\text { Continuity of care for patients } \\
\text { staying }>24 \mathrm{~h}\end{array}$ \\
\hline $\begin{array}{l}\text { Fewer barriers for entry into } \\
\text { observation care }\end{array}$ & $\begin{array}{l}\text { Continuity of contacts for } \\
\text { consulting specialists }\end{array}$ \\
\hline $\begin{array}{l}\text { Fewer handoffs for patients } \\
\text { staying }<24 \mathrm{~h}\end{array}$ & $\begin{array}{l}\text { Familiarity with longer-term } \\
\text { care coordination including } \\
\text { nursing home placement and } \\
\text { home health arrangements }\end{array}$ \\
\hline $\begin{array}{l}\text { Most observation unit studies } \\
\text { have been performed in unit } \\
\text { run by emergency medicine } \\
\text { physicians }\end{array}$ & \\
\hline
\end{tabular}

serial examination skills and enhance their diagnostic clinical judgment, the need for residents and their presumed opportunities for educational activities were ultimately greater in other areas of our hospital system that cared for patients with more acute illness; this led to the residents being taken off the observation service this year. Ample opportunity remains for medical students, interns, and residents to learn more about the care of such routine medical conditions as coronary artery disease, asthma, cellulitis, and heart failure by electively rotating through observation services.

It is important to understand that specialty consultations can be obtained if needed to care for patients on observation status. Relative value units and/or compensation are not dissimilar for the specialist seeing an observation patient versus a true inpatient. Many units function as a result of close collaboration between emergency medicine physicians or hospitalists and cardiologists. When calling a consultation for a patient on observation status it is important to express to the consultant that the patient's hospital stay is expected to be brief and that same-day consultation is requested.

Such allied health professionals as nurse practitioners or physician assistants may integrate well into an observation unit. The standardized care and less acute illness of patients in these units can facilitate the training of health professionals in standard and safe practices. Such practitioners can become experts in the management of diseases such as coronary artery disease and assume an important role of counseling patients thoroughly on their disease and its management at the time of discharge. The rapid turnover of patients in an observation unit with the obligatory documentation and counseling that accompanies these visits can make a unit with over ten beds challenging for one physician to staff. However, with the help of a well-trained allied health professional, one physician is likely to be able to care safely for all the patients in a 10-15 bed observation unit.

Nursing staff should be at least partially dedicated to the observation unit rather than the unit depending on a rotating nursing pool. The work flow can be different in an observation unit. Nursing assessments may be shorter than those on the inpatient wards but patient turnover is more brisk. The more comfortable nursing staff are with the standard operating procedures of an observation unit, the workflow there, and the diagnoses that are frequently seen, the more efficiently the unit will function. If nursing staff becomes expert in the most common diagnoses, patients obtain better care and counseling for these diseases than anywhere else in the hospital, and the unit can truly become a best-practice unit.

It is crucial to have an involved case manager who understands the intricacies of what constitutes observation versus inpatient status. Often an experienced case manager will have a better idea of these criteria than the physician in the unit, and the physician can turn to them for guidance. The case manager may also have to interact directly with insurance companies, so extended observation stays can be approved. Anecdotally some centers have had better success with patient selection for observation units if 24-h case management is available. Having an emergency department case manager screen admissions to see who is appropriate for observation status has proved to be the most effective way of avoiding patient and status misassignments for many hospitals.

Pharmacists working with observation patients should understand the policies governing limited reimbursement for oral medications provided to these patients. Pharmacists can take the lead in discussing non-crucial oral medications with the physicians caring for observation patients. Such steps could reduce unnecessary costs that get passed directly to the patient. Although reimbursement policies for observation patients seem to assume that patients will administer their own medications in this "outpatient setting", to protect patient safety many units insist on all medications coming from their hospital pharmacy.

Clerical staff should also be dedicated to the unit, because they can develop expertise in such tasks as ordering cardiovascular stress testing expediently after discharge. The importance of being able to arrange reliable follow-up should not be underestimated. Physicians in an observation unit are likely to be more comfortable discharging a patient after a short stay if are assured the patient will be seen for follow-up testing or evaluation in a matter of days. Many patients who are observed for chest pain may be candidates for outpatient stress testing within $72 \mathrm{~h}$ of discharge rather than waiting for inpatient testing. Good follow-up for conditions such as asthma or heart 
failure exacerbation may reduce the chances the patient will return to the hospital with persistent or recurrent symptoms.

An observation unit may provide an optimum environment for team-based care. Because these units are usually small to moderate-sized, and geographically defined, the providers working there have ample opportunity to get to know each other's work patterns and talents. Setting clear objectives (for example providing safe care for chest pain patients while keeping the median length of stay below $24 \mathrm{~h}$ ) can help all the practitioners in the unit develop a shared mental model of how patient care can best be provided in the unit. Because the number of diagnoses handled in an observation unit is limited, through repetition team members can become very good at working together to provide a safe, efficient, and standardized approach to patient care on the unit.

\section{Diagnoses Suitable for an Observation Unit}

By far the most tried and true use of an observation unit is to manage and risk-stratify patients who present to an emergency department with chest pain [3, 15]. Most centers use an algorithm to decide which patients are at low enough risk of cardiovascular morbidity and mortality to be cared for in an observation unit. Such algorithms vary by medical center but usually include chest pain that is controlled before transfer out of the emergency department: stable heart rate, blood pressure, and oxygen saturation, no signs of heart failure on examination, no new $\mathrm{Q}$ waves or ST segment elevation or depression on electrocardiogram, and a negative initial troponin measurement. Deciding on discharge within $24 \mathrm{~h}$ is usually straightforward for chest pain patients and is usually dependent on negative serial troponin measurements, sometimes coupled with noninvasive cardiac stress testing. Some centers struggle to organize cardiodiagnostic testing out-of-hours or during the weekend. If such arrangements cannot be made within the hospital a protocol by which patients can be discharged with an outpatient stress test scheduled within $72 \mathrm{~h}$ can be made with input from the local cardiology leadership team.

Data suggest that asthma can be treated in an emergency department observation unit at lower cost and with similar outcomes to inpatient admission [16]. Approximately $80 \%$ of patients who fail to improve after $4 \mathrm{~h}$ of emergency department therapy for an acute asthma exacerbation can be discharged after $12 \mathrm{~h}$ of continuous therapy [16]. Most asthma observation protocols have inclusion and/or exclusion criteria and discharge criteria at least partially based upon peak expiratory flow rates. Interestingly, patients treated in an observation unit rather than admitted to the hospital for an asthma exacerbation may score higher on quality-of-life indicators after discharge [16]. This may be secondary to a shorter stay leading to fewer psychological effects and disruption of patients' social roles outside the hospital.

A single-center study investigated the effects of evaluating syncope in an emergency department observation unit [17]. Patients admitted to the observation unit were those believed to be at intermediate risk of cardiovascular morbidity and mortality (high-risk patients were admitted directly to the hospital and low-risk patients were discharged from the emergency department). Using an observation unit for initial workup of intermediate-risk patients led to a vast reduction in total patient-hospital days. A presumptive diagnosis of the cause of syncope was established for two-thirds of patients evaluated in the observation unit [17]. However, it is important to note that the diagnostic evaluations in this unit were dependent on the ability to obtain expedient tilt-table testing, carotid massage with continuous beat-to-beat heart rate and blood pressure monitoring, and consultation from electrophysiologists.

Observation of hemodynamically stable patients with recent-onset (less than $48 \mathrm{~h}$ ) atrial fibrillation has also been examined in a single-center prospective, randomized trial [18]. A shortened median length of stay was demonstrated with use of the observation unit (10 vs. $25 \mathrm{~h}$ ) with no differences in adverse outcomes detected in the six months after discharge. It should be recognized that the observation protocol required procedural sedation and cardioversion to be provided by an emergency medicine attending physician for patients who had not spontaneously converted to sinus rhythm after $6 \mathrm{~h}$ of pharmacologic rate control and continuous cardiac monitoring.

Select patients with transient ischemic attacks may also be candidates for monitoring and workup in an observation unit. A single-center prospective randomized study found that pursuing an accelerated diagnostic protocol for transient ischemic attack patients observed in an emergency department unit could cut the duration of hospital stay and the cost of care by approximately one half, with clinical outcomes similar to those for patients admitted to the hospital [19]. The median length of stay for patients cared for in the observation unit was $26 \mathrm{~h}$. The algorithm used required every patient to receive serial clinical evaluations, neurology consultation, carotid Doppler, echocardiography and cardiac monitoring. The authors of this study attribute some of its success to "institutional commitment of resources to provide prompt services [19]."

Data also exist showing some patients with heart failure may be treated successfully in observation units [20]. Many other diseases are treated in observation units around the country, including cellulitis, gastroenteritis with dehydration, mild pyelonephritis, uncomplicated acute pain episodes in patients with sickle cell disease, low-risk upper 
Table 2 Diagnoses treated in observation units

\begin{tabular}{ll}
\hline $\begin{array}{l}\text { Diagnoses classically treated in } \\
\text { observation units }\end{array}$ & $\begin{array}{l}\text { Diagnoses sometimes treated in } \\
\text { observation units }\end{array}$ \\
\hline Chest pain & $\begin{array}{l}\text { Acute gastroenteritis } \\
\text { Asthma }\end{array}$ \\
Mickle cell acute pain episode \\
Transient ischemic attack & Low-risk upper GI bleed \\
Low-risk syncope & Mild alcohol withdrawal \\
& Mild community-acquired \\
& pneumonia \\
& Cellulitis \\
& Pyelonephritis without sepsis \\
\hline
\end{tabular}

gastrointestinal hemorrhage, and community-acquired pneumonia with a risk class of less than IV (Table 2). There are fewer data on the median length of stay, cost, and outcome of observing patients with these diagnoses. As part of the efficiency of an observation unit comes through treating patients with a limited set of diagnoses so that nurses and doctors can develop a standardized yet systematic approach, great consideration should be given to starting with a finite number of diagnoses when first opening an observation unit. The number of diagnoses can always be expanded later if patient volume remains too low for the number of beds designated for an observation unit. Selecting patients that are appropriate for an observation unit can be facilitated by using standardized inclusion and exclusion criteria (three examples are given in the Appendix).

\section{Financial Considerations}

Providing care to a patient under observation status has financial implications for the clinician, institution, and patient. The financial implications to the clinician are likely to be the least significant and relate to the relative value units that can be claimed with an observation visit compared with an inpatient visit. A high-level observation admission (99220) is worth 3.56 work relative value units (wRVUs) whereas a high-level inpatient admission (99223) is worth 3.86 wRVUs [21]. A high-level observation follow-up visit (99226) equates to the same 2.0 wRVUs as a high-level inpatient follow-up visit (99233) [21].

The financial considerations at the hospital level are much more substantial. The experience of the University of Wisconsin is provocative. For their general medicine observation encounters there was a net per-encounter loss of $\$ 1,378$ per stay which was calculated to be a loss of $\$ 33.53$ per hour of observation [8••]. It is important to realize that the patients analyzed in this study were admitted to the standard inpatient wards rather than to a dedicated observation unit. An argument for forming dedicated observation units is that care in such units costs less because it is protocol-based and minimizes the sometimes expensive differences between testing and length of stay for a given condition [22••]. Even if the care delivered to individual patients in an observation unit is not profitable, hospitals could minimize losses on observation patients by providing them structured and expedient yet safe care in an observation unit. With the expansion of the Medicare Recovery Audit Contractor program, observation stays are probably here to stay and hospitals need to develop a strategy for providing observation care in a manner that is both safe and economical. Having an observation unit may contribute to a hospital's financial health in indirect ways. Observation rooms are often smaller than inpatient rooms, enabling a hospital to care for more patients within its space constraints. With patient care often being more efficient in a dedicated observation unit, "virtual capacity" can be increased by caring for more patients (who are treated and discharged expediently) with the same number of beds [22••, 23]. Although creating "virtual capacity" is more important for hospitals running at capacity, less busy hospitals may also benefit from forming a dedicated observation unit. Staffing requirements are often lower in observation units, because patients have less acute illness. Therefore, by consolidating observation patients into a single unit, hospitals may save money on overall staffing costs (medical and surgery floors are usually staffed in a standard ratio without taking observation patients into account).

Patients can experience substantial financial burdens after an observation stay. Much has been made of this burden in both the popular press $[24,25]$ and academic journals [6•, 26•] within the last year. Observation is regarded as an "outpatient service" so it is reimbursed via Medicare part B and patients are responsible for a $20 \%$ copayment for professional and facility charges [26•]. Medicare will not pay for medications given while the patient is under observation that the patients could theoretically self administer, so these pharmacy charges may be passed directly to patients. Finally the time a patient spends in observation does not currently count toward the three midnights that a patient must stay in a hospital in order for Medicare to reimburse for the patient's subsequent skilled nursing facility stay. When hospital, pharmacy, and nursing home charges are totaled, patients may end up being responsible for payments that greatly exceed what they would have needed to pay for an inpatient stay [26•]. Because many patients are responsible only for their Medicare part A deductible after an inpatient stay and do not have to pay another deductible for 60 days after discharge, patients repeatedly admitted to an observation unit could have more out-of-pocket costs over time than patients who experience repeated inpatient admissions. Keeping in mind the financial risks to patients under observation care, it is prudent for the clinician providing observation services to minimize unnecessary hours in the hospital setting, 
low-yield imaging studies, and medications that are of questionable benefit. Units could provide the patient with Medicare's brochure on observation status at the time care is first rendered so they can begin to understand their financial obligations [27]. Providers should also be honest with patients that nursing facility stays would not be covered by Medicare after an observation stay; this could affect their decision on where to rehabilitate.

\section{Metrics for an Observation Unit}

The care provided in any observation unit should be regularly monitored to make sure that established clinical practice guidelines are being met for the diagnoses treated. Patient experience and satisfaction should be monitored through the Hospital Consumer Assessment of Healthcare Providers and Systems (HCAHPS) [14]. The medical director of the unit should monitor diagnoses for which patients are most commonly being admitted to the unit with and the average and median lengths of stay per diagnosis. Such data may help detect delays to care in specific realms such as diagnostic testing that may be remediated. Such data may also reveal that the local observation unit is not a suitable environment for some diagnoses. The conversion to inpatient admission from the observation unit should be monitored. Traditional teaching is that approximately $20 \%$ of patients under observation should eventually be admitted. If the actual number is lower, the patient population being observed may be too well and more of these patients should be discharged directly from the emergency department rather than being observed. If the number admitted is higher, patients being admitted to the observation unit are probably more ill than typical observation patients should be. Readmission should be monitored closely. Finally, a hospital's waiting times for initial evaluation in the emergency department and the number of patients who leave without being seen should be monitored, because an efficient observation unit may improve these metrics.

\section{Conclusion}

Although "observation status" remains confusing even to experts in the field, creating an observation unit with a focus on caring for patients with specific diagnoses expediently yet safely may improve hospital bed capacity and reduce overall healthcare costs. Although more hospitals are creating such units, only approximately a third of hospitals currently have one. As new units are established, careful consideration must be made of the needs of the local patient population. The number of beds assigned should be based upon the calculated need by patients with defined diagnoses, for which pre-specified treatment algorithms can be closely followed. A physician should be selected to lead a new observation unit on the basis of diverse factors, and the decision is best made at a local level. When a unit is established, a system of continuous quality improvement must be introduced by senior physicians and nurses, and standard metrics must be monitored regularly.

\section{Compliance with Ethics Guidelines}

Conflict of Interest Jason D. Napolitano and Inderpreet Saini declare no conflicts of interest.

Human and Animal Rights and Informed Consent This article does not contain any studies with human or animal subjects performed by any of the authors.

\section{Appendix}

Sample Triage Criteria \#1

Sickle Cell Anemia with Uncomplicated Acute Pain Episode

\section{Criteria for Observation}

Inclusion criteria:

1. Known diagnosis of sickle cell disease or sickle cell variant

2. Presenting complaint is pain

3. Pain relief not adequate with a reasonable trial of narcotic analgesics in the outpatient setting or the Emergency Department

Exclusion criteria:

1. New infiltrate on chest X-ray

2. New hypoxemia or increased oxygen requirement if on chronic $\mathrm{O}_{2}$

3. New focal neurologic findings

4. Expected need for exchange transfusion

5. Suspected splenic or hepatic sequestration crisis

6. Suspected aplastic crisis

7. Altered mental status

8. Acute renal failure

9. Chronic kidney disease with need to initiate dialysis

10. Sickle cell anemia with severe asthma exacerbation

11. Ongoing myocardial ischemia

12. Sickle cell anemia with sings and/or symptoms suggestive of acute left or right heart failure

13. Acute liver failure

14. Decompensated chronic liver failure

15. Acute cholecystitis or ascending cholangitis

16. New diagnosis of osteomyelitis

17. New diagnosis of avascular necrosis 
Sample Triage Criteria \#2

\section{Asthma}

\section{Inclusion Criteria for Observation Unit}

PEF $33-75 \%$ of best or predicted after initial ED treatments

PEF $>75 \%$ best or predicted but:

- Respiratory rate $>25$, or

- Pulse $>110$, or

- Cannot complete a sentence in one breath, or

- Pt does not have acceptable air movement or has severe wheezing on clinical exam, or

- Pt's symptom resolution and PEF improvement lasts for only a short period of time after each treatment, or

- $\mathrm{SaO}_{2}<95 \%$ or pt's known baseline

\section{Asthma}

\section{Exclusion Criteria for Observation Unit}

- PEF $<33 \%$ of best or predicted after initial ED management and/or treatment

- Pt with asthma and signs and/or symptoms of concomitant active medical illness (infiltrate on CXR suggestive of PNA, suspicion of CHF based on history, clinical exam, or BNP $>100$, etc.)

- Any features of life-threatening asthma including:

1. $\mathrm{SpO}_{2}<90 \%$ on room air

2. Silent chest

3. Cyanosis

4. Signs of fatigue/poor respiratory effort

5. Bradycardia

6. Arrhythmia

7. Relative hypotension

8. Exhaustion, confusion, or coma

9. $\mathrm{PaCO}_{2}>42 \mathrm{~mm} \mathrm{Hg}$ (note: $\mathrm{ABG}$ not required before admission in clinically stable pts)

$\mathrm{PEF}=$ Peak expiratory flow

\section{Sample Triage Criteria \#3}

\section{Acute Gastroenteritis/PO Intolerance/Dehydration}

\section{Observation Unit Inclusion Criteria}

- Dehydration with orthostatic hypotension or tachycardia

- Cause thought to be reversible within 24 h, i.e. viral or bacterial gastroenteritis
- Inability to tolerate crucial PO meds

Exclusion criteria:

- Bloody emesis

- Hematochezia with falling hematocrit

- Sodium $<125$

- Severe acute renal failure not likely to resolve with hydration (FeNa suggestive of intrinsic renal damage, etc.)

- Bicarbonate $<12$ on chemistry panel

- Anion gap $>15$

- Impending shock

\section{References}

Papers of particular interest, published recently, have been highlighted as:

- Of importance

•- Of major importance

1. Medicare Benefit Policy Manual. Chapter 6-Hospital Services Covered Under Part B. 2013. http://www.cms.gov/Regulationsand-Guidance/Guidance/Manuals/downloads/bp102c06.pdf. Accessed 17 Oct 2013.

2. Leykum LK, Huerta V, Mortensen E, et al. Implementation of a hospitalist-run observation unit and impact on length of stay (LOS): a brief report. J Hosp Med. 2010;5:E2-5.

3. Roberts RR, Zalenski RJ, Mensah EK, et al. Costs of an emergency department-based accelerated diagnostic protocol versus hospitalization in patients with chest pain: a randomized controlled trial. JAMA. 1997;278:1670-6.

4. Rydman RJ, Isola ML, Roberts RR, et al. Emergency department observation unit versus hospital inpatient care for a chronic asthmatic population: a randomized trial of health status outcome and cost. Med Care. 1998;36:599-609.

5. Decker WW, Smars PA, Medano P, et al. A prospective randomized trial of an emergency department observation unit for acute onset atrial fibrillation. Ann Emerg Med. 2008;52:322-8.

6. - Wachter RM. Observation status for hospitalized patients. JAMA Intern Med. 2013;173(21):1999-2000. This opinion piece provides a great summary of the current challenges providers and patients are finding in the current Medicare regulations on observation status.

7. Jaffe S. Seniors sue medicare to close nursing home coverage gap. http://chit.org/2013/05/03/seniors-sue-medicare-to-closenursing-home-coverage-gap/. Accessed 25 Oct 2013.

8. - Sheehy AM, Graf B, Gangireddy S et al. Hospitalized but not admitted characteristics of patients with "observation status" at an Academic Medical Center. JAMA Intern Med. 2013; 173(21):1991-1998. This article is a retrospective review of the patients admitted to an academic medical center between July 2010 and December 2011. It is notable in that it demonstrates just how much "observation status" has changed from the way it was originally defined by Medicare. Patients are now being observed for a vast array of diagnoses and the length of stay in observation is increasing dramatically. 
9. Jaffe S. FAQ: hospital observation can be poorly understood and costly for medicare beneficiaries. http://www.kaiserhealthnews. org/stories/2013/September/04/observation-care-faq.aspx. Accessed 17 Oct 2013.

10. Jackson Davis HealthCare. The medicare recovery audit contractor (RAC) program: an evaluation of the 3 years demonstration. http://www.racaudits.com/uploads/RAC_Demonstration_ Evaluation_Report.pdf. Accessed 25 Oct 2013.

11. Wachter, B. Observation status: how medicare's solution could make things worse. http://www.kevinmd.com/blog/2013/08/observ ation-status-medicares-solution-worse.html. Accessed 25 Oct 2013.

12. - Wiler JL, Ross MA, Ginde AA. National study of emergency department observation services. Acad Emerg Med. 2011; 18(9):959-965. This paper gives a good overview as to where US hospitals are as far as adoption of dedicated observation units.

13. Liudvikas J, Partridge R. A comparison of emergency department versus in hospital chest pain observation units. Am J Emerg Med. 2005;23(2):111-3.

14. Green A, Barsuk J, Casey DE et al. The observation unit: an operational overview for the hospitalist. A SHM white paper accessed at: http://www.hospitalmedicine.org/AM/Template.cfm? Section=White_Papers\&Template=/CM/ContentDisplay.cfm\& ContentID=21890. Accessed 25 Oct 2013.

15. Gomez MA, Anderson JL, Karagounis LA, et al. An emergency department-based protocol for rapidly ruling out myocardial ischemia reduces hospital time and expense: results of a randomized study (ROMIO). J Am Coll Cardiol. 1996;28:25-33.

16. Rydman R, Isola M, Roberts R, et al. Emergency department observation unit versus hospital inpatient care for a chronic asthmatic population: a randomized trial of health status outcome and cost. Med Care. 1998;36(4):599-609.

17. Shen WK, Decker WW, Smars PA, et al. Syncope evaluation in the emergency department study (SEEDS): a multidisciplinary approach to syncope management. Circulation. 2004;110: 3636-45.

18. Decker WW, Smars PA, Vaidyanathan L, et al. A prospective randomized trial of an emergency department observation unit for acute onset atrial fibrillation. Ann Emerg Med. 2008;52:322-8.
19. Ross MA, Compton S, Medado P, et al. An emergency department diagnostic protocol for patients with transient ischemic attack: a randomized controlled trial. Ann Emerg Med. 2007;50: 109-19.

20. Peacock WF, Remer EE, Aponte J, et al. Effective observation unit treatment of decompensated heart failure. Congest Heart Fail. 2002;8:68-73.

21. Mahrous T. Work (wRVU) and total (tRVU) list for hospitalist E/M CPT ${ }^{\circledR}$ codes. http://thehappyhospitalist.blogspot.com/2011/ 02/rvu-em-2011-work-wrvu-and-total-trvu.html. Accessed 14 Oct 2013.

22. •• Baugh CW, Venkatesh AK, Hilton JA et al. Making greater use of dedicated hospital observation units for many short stay patients could save $\$ 3.1$ billion a year. Health Aff (Millwood). 2012;31(10):2314-2323. This article uses a simulation model to estimate national cost savings if all hospitals used efficientlyfunctioning dedicated observation units.

23. Roberts R, Graff LG. Economic issues in observation unit medicine. Emerg Med Clin North Am. 2001;19(1):19-33.

24. Graham J. When you're not a hospital inpatient: observation care. Chicago Tribune. 2009.

25. Span P. In the hospital but not really a patient. New York Times. (http://newoldage.blogs.nytimes.com/2012/06/22/in-the-hospitalbut-not-really-a-patient/). Accessed 22 Jun 2012.

26. - Baugh CW, Schuur JD. Observation care- High-value care or a cost-shifting loophole? N Eng J Med 2013;369:302-305. Viewing the table on hospital costs for a theoretical observation status patient in the paper can help provide an understanding of what patient's bill might look like after receiving observation-level care.

27. Centers for Medicare \& Medicaid Services. Are you a hospital inpatient or outpatient? If you have Medicare ask! 2013. www. medicare.gov/pubs/pdf/11435.pdf. Accessed 25 Oct 2013. 